\title{
8. Cardiología oncológica
}

\subsection{Mixomas cardiacos: reporte de 6 casos}

Durán Sáinz Víctor Manuel,

Soto González Juan Ignacio, García Jiménez Yoloxóchitl, Hernández Bravo Mariana Jazmín, Mariscal Uriarte Javier, Medina Gómez Héctor Alejandro

Hospital de Especialidades UMAE No. 14, IMSS, Veracruz.

Tipo de estudio: Reporte de casos clínicos con revisión de fuentes

Introducción: El mixoma ocupa de 30 a 50\% de los tumores primarios de corazón. Su localización en la aurícula izquierda es de 75\% y en la derecha de $15-20 \%$. La edad media de diagnóstico son los 50 años y aproximadamente $70 \%$ son mujeres. El ecocardiograma transtorácico (ECOT) permite realizar un análisis del tumor. Descripción del caso: Entre agosto de 2018 y febrero de 2020 seis pacientes fueron operados por mixoma cardiaco, previo protocolo por ECOT y coronariografía. El síntoma predominante fue disnea, y en un caso embolismo cerebral. La edad promedio fue 57 años, una proporción de sexo 2:1 (mujeres:hombres). Predominó el mixoma auricular izquierdo (cuatro pacientes). Se realizó resección en todos los casos, con evolución postquirúrgica satisfactoria. Metodología: Se realiza una revisión sistemática y exhaustiva de todos los artículos indexados de 2016 a 2020 disponibles en la base de datos de PubMed-Medline implementando las palabras claves: myxoma, cardiac tumor, dando relevancia al factor de impacto. Resultados: La presentación clínica de estos seis casos estuvo asociada a su localización predominantemente izquierda; las características de los pacientes fueron similares a lo reportado en la literatura, prevaleciendo el género femenino y la edad promedio de presentación. La evaluación por ECOT como primera modalidad de imagen permite un diagnóstico oportuno por su disponibilidad, bajo costo y caracterización del tumor. Discusión: Aunque el mixoma es el tumor benigno más común en el adulto, sigue siendo una entidad rara ( $0.25 \%$ de las enfermedades cardiacas). El diagnóstico a través de estudios no invasivos como el ECOT favorece una atención inmediata que reduce las complicaciones. Además, permite diferenciar con otras masas intracardiacas y en el seguimiento postquirúrgico, dada la recurrencia hasta de $15 \%$.

\subsection{Fibrosarcoma cardiaco, reporte de un caso}

Macías Argüelles Dafne Muriel,* Vargas Ascencio Paulo Iván, ${ }^{*}$ Flores García Carlos, ${ }^{\ddagger}$ Díaz Díaz Enrique ${ }^{\ddagger}$

* Instituto Mexicano del Seguro Social. ^ Hospital de Cardiología del Centro Médico Nacional Siglo XXI, IMSS.

Tipo de estudio: Reporte de casos clínicos con revisión de fuentes

Introducción: El fibrosarcoma es un tumor mesenquimal maligno, representan entre cinco y $10 \%$ de los sarcomas de tejidos blandos. Los tumores cardiacos primitivos son infrecuentes, su incidencia aproximada es de un caso por cada 100,000 habitantes/año. Los tumores primitivos malignos representan alrededor de $25 \%$ de los tumores cardiacos y dentro de ellos los sarcomas son los más habituales. Descripción del caso: Mujer de 49 años, disnea de esfuerzo de tres meses de evolución; soplo diastólico mitral. Ecocardiograma muestra

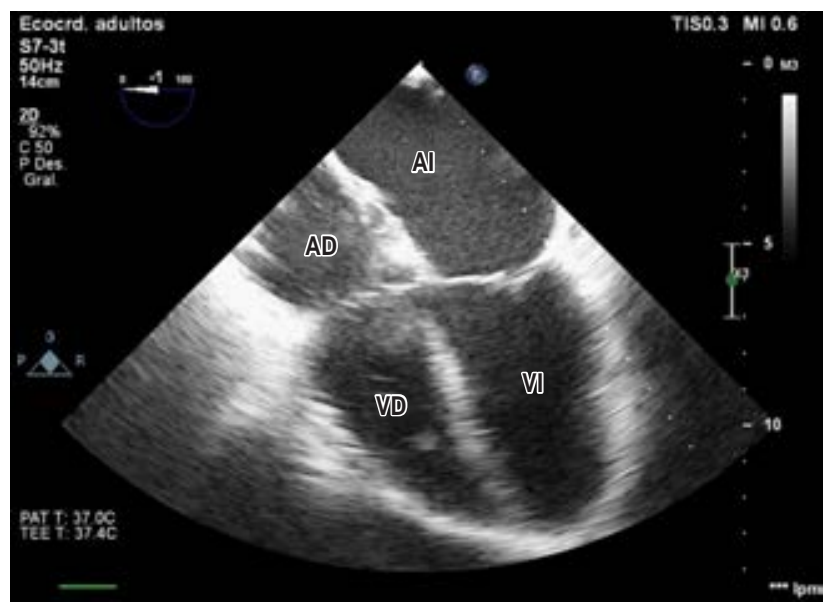

Figura 8.1.2: Ecocardiograma transesofágico posterior a resección quirúrgica de mixoma auricular izquierdo.
Figura 8.1.1:

A) Tumoración de aurícula izquierda con características de mixoma que obstruye la válvula mitral y protruye en ventrículo izquierdo. B) Pieza anatomopatológica del mixoma descrito. Medidas $5.3 \times 3.7 \mathrm{~cm}$.
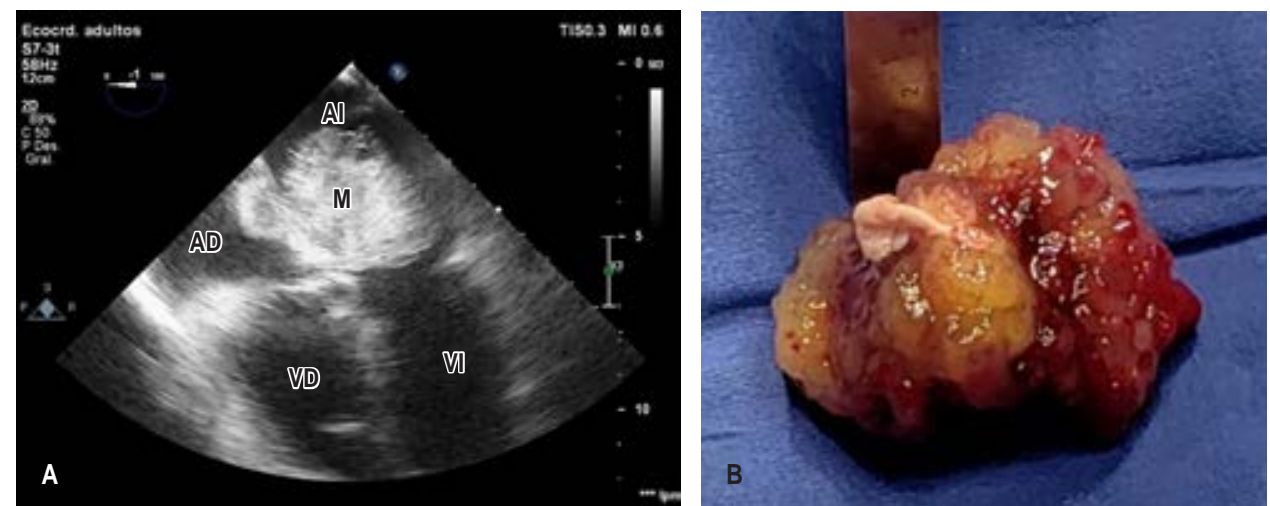

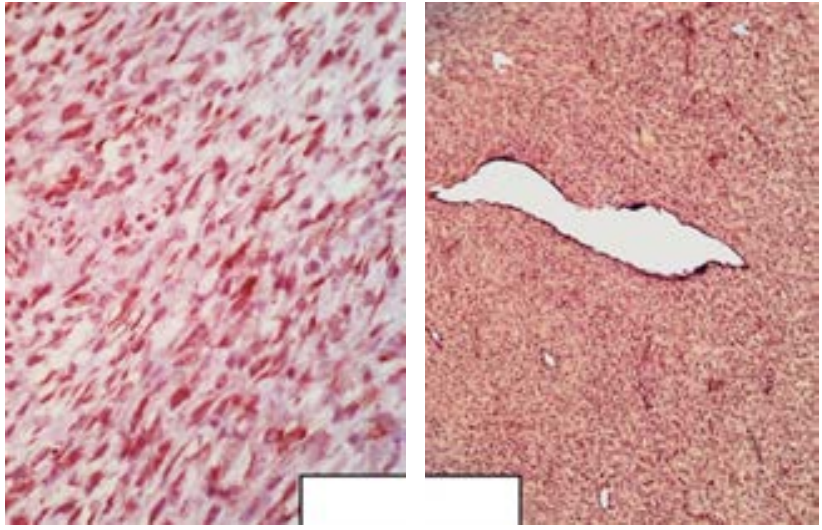

Figura 8.2.1.
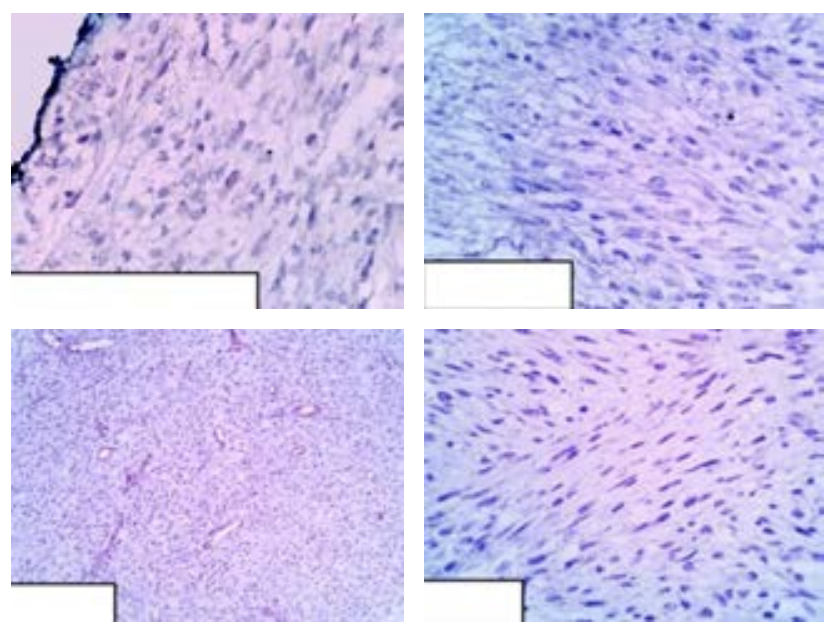

Figura 8.2.2.

masa adosada en fosa oval de $42 \times 33 \mathrm{~mm}$, gradiente mitral 25/14 $\mathrm{mmHg}$, THP $230 \mathrm{mseg}$, velocidad de orejuela izquierda $34 \mathrm{~cm} / \mathrm{seg}$, PSAP 50 mmHg, FEVI 60\%. Metodología: Debido a hallazgos ecocardiográficos y sintomatología se realizó resección de tumor intraauricular más reparación de septum interauricular; diagnóstico histopatológico: fibrosarcoma de $5.5 \mathrm{~cm}$, borde quirúrgico positivo para neoplasia. En seguimiento por oncología. Resultados: Representa menos de 5\% de los sarcomas cardiacos. Cualquier localización cardiaca. La sintomatología depende del tamaño y la localización: síntomas cardiovasculares, embolismo, síndrome constitucional. Ecocardiográficamente: bordes irregulares, poco móviles, base amplia; ante sospecha realizar TAC o/y RMN, metástasis con gammagrafía. Diagnóstico definitivo por biopsia: células en huso, núcleos romos, citoplasma alargado, abundantes mitosis, escaso pleomorfismo y anaplasia; inmunohistoquímica: vimentina y colágeno tipo I. Discusión: La conducta terapéutica dependerá de la prontitud con la que se logre establecer el diagnóstico. El tratamiento es quirúrgico y consiste en la resección del tumor, adicional a radioterapia y quimioterapia según estadio. Independientemente del grado, la tasa de supervivencia general a cinco años es de alrededor de 40-60\%.

\subsection{Mixoma auricular derecho: reporte de caso}

Pérez Roa Herlich Francisco,* Soto González Juan Ignacio, ${ }^{\ddagger}$ Medina Gómez Héctor Alejandro, ${ }^{\ddagger}$ Mariscal Uriarte Javier, ${ }^{\ddagger}$ Hernández Bravo Mariana Jazmín, ${ }^{\ddagger}$ Durán Sáinz Víctor Manuel, ${ }^{\ddagger}$ Sánchez Martínez María Isabel ${ }^{\ddagger}$

* Instituto Mexicano del Seguro Social. ‡ Hospital de Especialidades UMAE No. 14, IMSS, Veracruz.

Tipo de estudio: Reporte de casos clínicos con revisión de fuentes

Introducción: De acuerdo con la patología el 45\% de los tumores benignos son mixomas; éstos son masas intracardiacas comúnmente encontradas en la aurícula izquierda, pero puede ser vista en la aurícula derecha raramente. La edad media de diagnóstico son los 50 años y aproximadamente $70 \%$ son mujeres. El ecocardiograma transtorácico (ECOT) puede orientar para diferenciar el diagnóstico entre tumores benignos y malignos. Descripción del caso: Femenino de 58 años, presenta disnea progresiva, dos eventos de síncope y edema inferior; a la exploración retumbo diastólico tricuspídeo. ECOT: tumor atrial derecho $(43 \times 39 \mathrm{~mm})$ (Figura 8.3.1); angiografía: coronarias sin lesiones, imagen pediculada con vascularidad proveniente de coronaria derecha (Figura 8.3.2). Amerita cirugía intracardiaca para resección de tumor de $45 \times 40$ mm de diámetro. Reporte histopatológico: mixoma cardiaco. Metodología: Se realiza una búsqueda sistemática y exhaustiva de todos los artículos indexados de 2016 a 2020 disponibles en la base de datos PubMedMedline implementando las palabras clave: myxoma, cardiac tumor, dando relevancia al factor de impacto. Resultados: Se reporta al ECOT como primera modalidad de imagen por disponibilidad, bajo costo y portabilidad. Se documenta incidencia de $2 \%$ de mixomas auriculares derechos, por lo que es de interés epidemiológico debido a los datos clínicos de insuficiencia cardiaca derecha. El seguimiento con ECOT es recomendado como mínimo por cuatro años por el aproximado de 10 a 15\% de recurrencia del tumor. Discusión: Al considerar la escasa incidencia de los mixomas en población adulta es de relevancia la presentación de una neoplasia auricular derecha por ser una entidad más atípica, con presentación de interés por síncope y disnea, logrando su diagnóstico oportuno por ECOT y resolución quirúrgica satisfactoria.

\subsection{Angiosarcoma auricular izquierdo}

Capi Barradas Daniel Alejandro, Rico Rico José Luis Instituto Mexicano del Seguro Social.

Tipo de estudio: Reporte de casos clínicos con revisión de fuentes

Introducción: Los tumores intracardiacos tienen una prevalencia de $0.001-0.03 \%$. De los sarcomas, el angiosarcoma representa $76 \%$, predomina del lado derecho, se presenta con pico de edad a los 40 años, pero va desde uno a 76 años. Se requiere resección quirúrgica y quimioterapia con doxorrubicina e ifosfamida. El pronóstico va de los seis a los 25 meses. El ecocardiograma posee una sensibilidad de $100 \%$ para valorar cavidad-movilidad tumoral. La RMN detalla el microambiente-composición tumoral. Descripción del caso: Masculino de 20 años. Un mes con disnea progresiva a pequeños esfuerzos. TC de tórax: tumoración intracardiaca en aurícula izquierda. Ecocardiograma: tumoración intraauricular izquierda de $77 \times 64 \times 54 \mathrm{~mm}$, protruye a VI en diástole. Gradiente transmitral $17 \mathrm{mmHg}$, PSAP: $60 \mathrm{mmHg}$. Foramen oval flujo Doppler hacia AD. Índice VD/VI: 1.1. Durante la cirugía cardiotorácica se encuentra tumoración intrauricular de $8 \times 6.7 \times 5.5 \mathrm{~cm}$, pedi- 


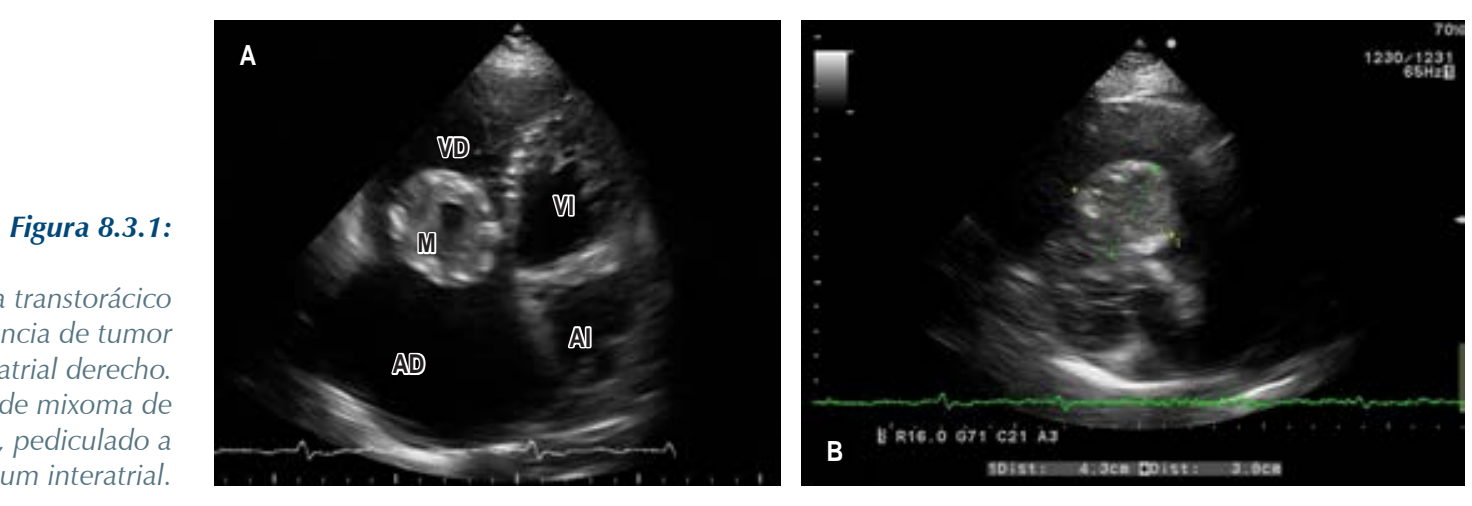

A) Ecocardiograma transtorácico con evidencia de tumor intracardiaco atrial derecho.

B) Medición de mixoma de

$43 \times 39 \mathrm{~mm}$, pediculado a expensas de septum interatrial.

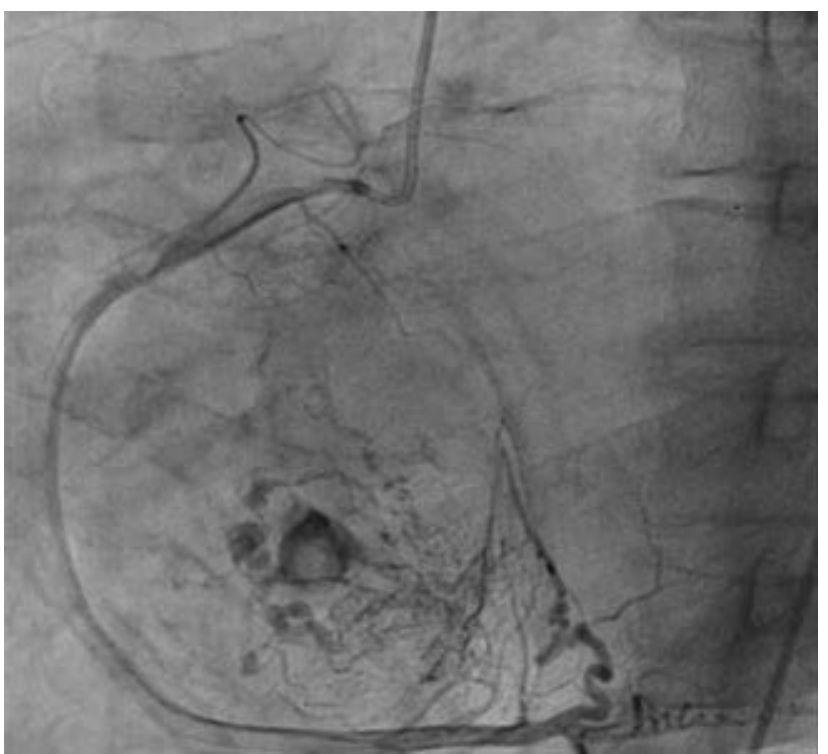

Figura 8.3.2: Cateterismo cardiaco con evidencia de imagen pediculada con vascularidad proveniente de coronaria derecha.

culado borde posterior. Sangrado mayor al habitual, hipotensión y paro cardiorrespiratorio sin respuesta. Metodología: A través del método booleano se realizó búsqueda de términos MESH «atrial» [and] «tumor» en las bases de datos con repositorios de evidencia científica arbitrada indizada (PubMed) y no indizada (Google Scholar). Resultados: El angiosarcoma auricular, a pesar de ser el tumor maligno más frecuente del lado derecho del corazón, cuenta con relevancia dentro de los tumores intracardiacos, ya que el resto representan una prevalencia ínfima. Amerita tratamiento quirúrgico, el cual — a pesar de la coadyuvancia con quimioterapia — revela un porcentaje muy reducido de supervivencia en el primer año desde el diagnóstico. Discusión: Los tumores intracardiacos se presentan de manera clásica en el lado derecho del corazón, siendo este caso una excepción interesante a dicha tendencia. Cobran especial relevancia los estudios de imagen, los cuales ayudan en el diagnóstico oportuno y en el análisis histopatológico, el cual permite la adecuada caracterización de la lesión.
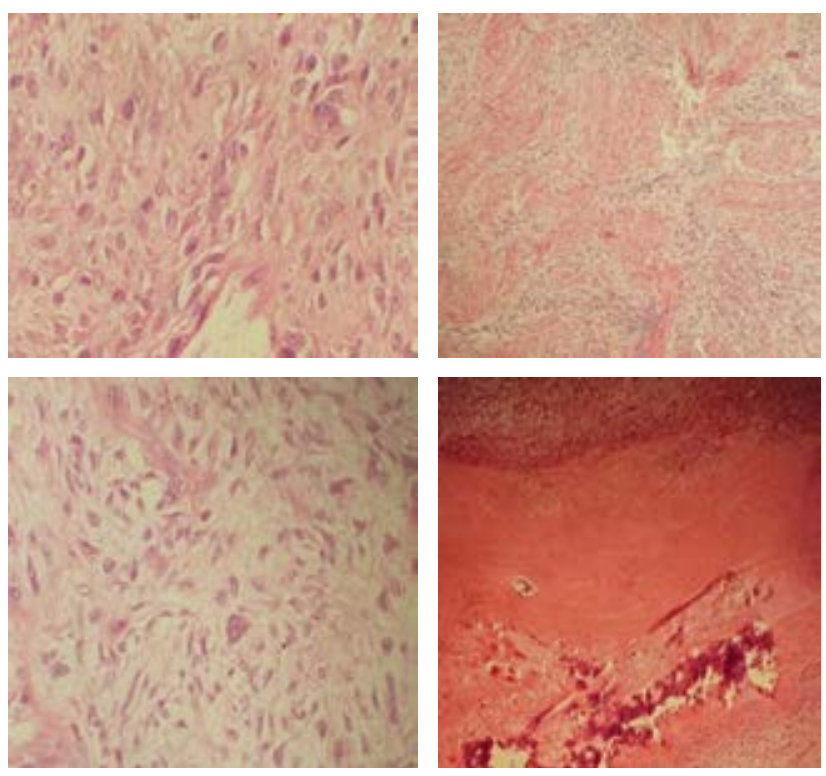

Figura 8.4.1. 